\title{
Estimativa de parâmetros técnicos da tecnologia de aplicação do glyphosate no controle de Brachiaria decumbens ${ }^{1}$
}

\author{
Renato Adriane Alves Ruas ${ }^{2}$, Mauri Martins Teixeira ${ }^{3}$, Antônio Alberto da Silva, \\ Haroldo Carlos Fernandes ${ }^{3}$, Rogério Faria Vieira
}

\section{RESUMO}

A interação entre o diâmetro da mediana volumétrico (DMV), a densidade (DEN) e a cobertura (COB) proporcionada pelas gotas produzidas na pulverização, é pouco estudada e pode ter influência nas aplicações de herbicidas. Objetivouse estimar parâmetros técnicos da tecnologia de aplicação do glyphosate no controle de Brachiaria decumbens analisando os fatores que constituem a pulverização. Os tratamentos constaram da aplicação de glyphosate em diferentes modos de operação de um pulverizador centrífugo, visando a obtenção de parâmetros da aplicação: cinco rotações do dispositivo pulverizador $\left(105,157,210,262\right.$ e $\left.315 \mathrm{rad} \mathrm{s}^{-1}\right)$ e seis velocidades de deslocamento $(0,5 ; 1,0 ; 1,5$; 3,0; 4,5; e 6,0 $\mathrm{km} \mathrm{h}^{-1}$ ), obtendo-se trinta parâmentros. O herbicida foi aplicado aos 20 dias após a emergência (DAE). O pulverizador foi adaptado em barra equipada com motor elétrico e comando para controle da velocidade de deslocamento. Foram realizadas avaliações visuais e calculada a porcentagem de acúmulo de massa de matéria seca das plantas em relação às testemunhas, que não receberam herbicida. O ensaio foi conduzido em delineamento inteiramente casualizado, com cinco repetições, e os dados analisados por meio de regressão múltipla. Foram obtidos controles superiores a $91 \%$, quando o pulverizador foi utilizado de modo a proporcionar DMV de $327 \mu \mathrm{m}$ e COB 2,51\%, desde que a DEN fosse de 30 gotas $\mathrm{cm}^{-2}$. Os fatores que mais afetaram o controle de $B$. decumbens foram a cobertura e a densidade de gotas. A pulverização do glyphosate com menores DMV e COB e maiores DEN, proporcionou melhor controle das plantas.

Palavras-chave: Pulverização, planta daninha, agrotóxicos.

\section{ABSTRACT}

\section{Estimate of technical parameters of glyphosate application technology in the control of Brachiaria decumbens}

The interaction between the volumetric median diameter (VMD), density (DEN) and coverage (COV) provided by spray droplets is scarcely studied and may influence the application of herbicides in general. This study aimed to estimate the technical parameters of the glyphosate application technology in the control of Brachiaria decumbens Stapf. The treatments consisted of the combination of five rotational speeds of a centrifugal sprayer $(105,157,210,262$, $\left.315 \mathrm{rad} \mathrm{s}^{-1}\right)$ with six travel speeds $\left(0.5 ; 1.0 ; 1.5 ; 3.0 ; 4.5\right.$ and $\left.6.0 \mathrm{~km} \mathrm{~h}^{-1}\right)$. The tests were performed in the laboratory, and the sprayer was adapted into a bar equipped with an electric engine and a control unit to control the travel speed. Visual analyses were carried out and percentage of reduction in plant dry biomass was compared with the control without

Recebido para publicação em agosto de 2009 e aprovado em outubro de 2010

'Parte da tese de doutorado apresentada à Universidade Federal de Viçosa (UFV).

${ }^{2}$ Engenheiro-Agrônomo, Doutor. Universidade Federal de Viçosa, Campus de Rio Paranaíba, Rodovia BR 354, km 310, Caixa Postal 22, 38810-000, Rio Paranaíba, Minas Gerais, Brasil.renatoruas@ufv.br

${ }^{3}$ Engenheiro-Agrônomo, Doutor. Universidade Federal de Viçosa, Departamento de Engenharia Agrícola, Avenida P.H.Rolfs, s/n, Campus Universitário, 36570-000,Viçosa, Minas Gerais, Brasil. mauri@ufv.br, haroldo@ufv.br

${ }^{4}$ Engenheiro-Agrônomo, Doutor. Universidade Federal de Viçosa, Departamento de Fitotecnia, Avenida P.H.Rolfs, s/n, Campus Universitário, 36570-000, Viçosa, Minas Gerais, Brasil. aasilva@ufv.br

${ }^{5}$ Engenheiro-Agrônomo, Doutor. Embrapa/Epamig, Vila Gianet, casa 47, Campus Universitário, 36570-000, Viçosa, Minas Gerais, Brasil. rfvieira@epamig.br 
herbicide. The experiment was arranged in a completely randomized design, with five repetitions. Data were analyzed by multiple regression. Control of Brachiaria decumbens above $91 \%$ was obtained when the sprayer was used to provide VMD $327 \mu \mathrm{m}$ and COV of $2.51 \%$, with DEN at 30 droplets $\mathrm{cm}^{-2}$. The factors that most affected the control of $B$. decumbens were the coverage and droplet density. Spraying of glyphosate with lower VMD and COV and higher DEN provided better weed control.

Key words: Pesticides, spraying, weed.

\section{INTRODUÇÃO}

Uma das possíveis maneiras de se monitorar a qualidade das aplicações de agrotóxicos em geral, consiste em analisar a deposição das gotas em alvos artificiais, a fim de determinar o espectro e a população de gotas mais adequadas ao tratamento. Ao contrário do que se pode imaginar, a deposição das gotas sobre o alvo a ser controlado é complexa e pode afetar sobremaneira a eficácia dos tratamentos. A interação entre os parâmetros da aplicação como, por exemplo, tamanho das gotas produzidas, assim como a sua distribuição pelo alvo e a cobertura proporcionada pela aplicação, são fatores pouco estudados e que podem ter influência no controle, dependendo do tipo de produto utilizado e das características físicoquímicas do alvo.

Embora proporcione boa uniformidade de distribuição volumétrica, o processo hidráulico de produção de gotas de pulverização resulta em gotas de diversos tamanhos, podendo comprometer a eficácia dos tratamentos. De acordo com o Conselho Britânico de Proteção de Culturas (BCPC), as gotas com diâmetro inferior a $141 \mu \mathrm{m}$ são muito acometidas por deriva e evaporação e gotas com diâmetro superior a $800 \mu \mathrm{m}$, ao se chocarem contra o alvo, tendem a escorrer rapidamente para o solo e, assim, não há tempo suficiente para o produto ser absorvido pelo alvo biológico (Mirales \& Hewitt, 1997). Por essa razão, torna-se necessário maior gasto do produto para a compensação dessas perdas, a fim de garantir deposição da quantidade mínima adequada do produto sobre o alvo.

Venturelli et al. (2006) afirmaram que gotas uniformes, normalmente, proporcionam maior eficiência biológica pelo fato de transportar, aproximadamente, a mesma concentração do produto por gota, o que contribui para uma absorção mais uniforme do ingrediente ativo.

Dentre os equipamentos que produzem gotas uniformes, destaca-se o pulverizador centrífugo, cujo coeficiente de homogeneidade das gotas está, normalmente, próximo a 1,2, o que é considerado muito bom, pois, nesse caso, a maioria das gotas produzidas possui, aproximadamente, o mesmo diâmetro (Laguna, 2000).
Alguns trabalhos divergem quanto à hipótese do tamanho da gota produzida afetar ou não a eficácia do glyphosate. Merritt (1982) e Gebhardt et al. (1986) mostraram não haver efeito do tamanho da gota de glyphosate na eficácia dos tratamentos. Segundo Prasad \& Cadogan (1992), gotas pequenas podem proporcionar melhor controle na aplicação do glyphosate, pois apresentam boa cobertura das folhas das plantas e retêm o produto por mais tempo, favorecendo, assim, sua absorção. Liu et al. (1996), ao trabalharem com uma amplitude de diâmetros de gotas de 326 a $977 \mu \mathrm{m}$, observaram aumento na absorção do glyphosate à medida que aumentava o diâmetro das gotas, desde que a concentração do herbicida permanecesse constante. Venturelli et al. (2006) também trabalharam com diferentes tamanhos de gotas para aplicação de glyphosate. Eles verificaram maior eficácia do glyphosate quando as gotas produzidas possuíam diâmetros superiores a $250 \mu \mathrm{m}$, provavelmente devido ao fato de gotas pequenas terem sido acometidas por deriva.

Costa et al. (2008) avaliaram a eficácia do glyphosate aplicado com diversos diâmetros de gotas em volumes de calda que variaram de 100 a $200 \mathrm{~L} \mathrm{ha}^{-1}$ na dessecação de Brachiaria brizantha (Hochst. ex A. Rich.) Stapf. Todas as condições de aplicações foram eficientes, o que evidencia a possibilidade de redução do volume de aplicação e da dosagem do herbicida na dessecação de pastagens, considerando-se a utilização de herbicidas sistêmicos.

Pelo exposto, verifica-se que normalmente, o parâmetro da aplicação mais estudado é o tamanho das gotas produzidas. Porém, acredita-se que, estudado isoladamente, pode não explicar realmente os efeitos da pulverização. Portanto, objetivou-se estimar parâmetros técnicos da tecnologia de aplicação do glyphosate no controle de $B$. decumbens, analisando a combinação entre os fatores que constituem a pulverização.

\section{MATERIAL E MÉTODOS}

O experimento foi conduzido em casa de vegetação, e as aplicações do herbicida foram realizada no Laboratório de Mecanização Agrícola do Departamento de Engenharia Agrícola da Universidade Federal de Viçosa. 
Os tratamentos visando a obtenção dos parâmetros da aplicação, foram obtidos por meio de diferentes combinações de operação de um pulverizador centrífugo. Para isso, foram empregadas cinco rotações do dispositivo rotativo $\left(105,157,210,262\right.$ e $\left.315 \mathrm{rad} \mathrm{s}^{-1}\right)$ e seis velocidades de deslocamento do conjunto de pulverização $(0,5 ; 1,0$; 1,$5 ; 3,0 ; 4,5 ; \mathrm{e} 6,0 \mathrm{~km} \mathrm{~h}^{-1}$ ). Dessa forma, foram obtidos 30 diferentes parâmetros de diâmetro da mediana volumétrico (DMV), densidade (DEN) e cobertura (COB). Para cada condição de operação preparou-se previamente a calda de pulverização em galão de $20 \mathrm{~L}$, de modo que a dose do herbicida a ser aplicado fosse aquela inicialmente determinada, independentemente da variação da faixa útil de aplicação, velocidade de deslocamento ou rotação do pulverizador (Tabela 1).

O delineamento experimental utilizado foi o inteiramente casualizado, com 30 tratamentos, cada um constituído por diferentes espectros e populações de gotas, com cinco repetições, e cinco testemunhas, que não receberam herbicida, totalizando 155 unidades experimentais.

O herbicida utilizado nos tratamentos foi o glyphosate (sal de isopropilamina), na formulação CS com $360 \mathrm{~g} \mathrm{~L}^{-1} \mathrm{de}$ equivalente ácido aplicado aos 20 dias após a emergência (DAE) de plantas de B. decumbens. Empregou-se a dose de $150 \mathrm{~g} \mathrm{ha}^{-1}$ de glyphosate, definida previamente por meio de ensaio preliminar.

No intuito de uniformizar os tratamentos, sementes de $B$. decumbens foram colocadas para germinar em caixas de polietileno com areia. Em seguida, aleatoriamente, transplantaram-se quatro plântulas para vasos de polietileno contendo 3,5 L de solo, que foi classificado como Argissolo Vermelho-Amarelo Câmbico, com $42 \%$ de argila.

À época da aplicação, as plantas encontravam-se com aproximadamente $35 \mathrm{~cm}$ de altura e possuíam até dois perfilhos. Para aplicação do herbicida, foi utilizado um pulverizador centrífugo acionado por um motor elétrico de corrente contínua de 9 watts de potência, equipado com dispositivo rotativo horizontal cônico de $0,08 \mathrm{~m}$ de diâmetro e monitorado por um potenciômetro, que permitia variar sua rotação. O reservatório de calda do pulverizador possuía capacidade para $12 \mathrm{~L}$. O pulverizador cen- trífugo foi adaptado a uma barra de pulverização autodeslizante equipada com motor trifásico de $1 \mathrm{HP}$, sendo acionado por inversor de frequência vetorial modelo VFD-B, o qual possibilitava controlar o avanço e recuo do pulverizador em diferentes velocidades.

Para a determinação dos espectros e populações de gotas em cada condição de operação do pulverizador, ou seja, DMV, DEN e COB, empregaram-se etiquetas de papel contact branco, marca Vulcan, a fim de coletar imagens dos impactos das gotas. Utilizou-se o corante negro de marca Guarany, indicado para tintura de tecidos, na proporção de $10 \mathrm{~g} \mathrm{~L}^{-1} \mathrm{de}$ água para obter contraste na superfície amostradora. As imagens das etiquetas para análise foram obtidas, utilizando-se um "scanner" marca Hewlett Packard, modelo Scanjet 4 p, com resolução de 600 dpi. Cada grupo de etiquetas do mesmo tratamento, devidamente identificadas, foi escaneado e processado no programa computacional para análise de imagens “Image Tool", versão 2.0, calibrado com a imagem de um paquímetro escaneado da mesma forma das etiquetas e o programa Excel.

Utilizou-se a Equação 1 para a correção da dispersão das gotas na etiqueta de papel contact (Prat et al., 2006).

$\hat{y}=1,6454 x-12,33$

em que:

$\hat{y}=$ diâmetro da mancha, $\mu \mathrm{m} ; \mathrm{e}$

$\mathrm{x}=$ diâmetro da gota, $\mu \mathrm{m}$.

As aplicações foram realizadas em uma câmara especial para ensaios de pulverização. A temperatura e umidade relativa do ar no momento das aplicações variaram entre 17,5 e $18{ }^{\circ} \mathrm{C}$ e 80 e $85 \%$, respectivamente.

Para avaliação da eficácia do glyphoste no controle das plantas, foram realizadas análises visuais e porcentagem de acúmulo de massa de matéria seca em relação às testemunhas.

As avaliações visuais foram realizadas aos 7, 14, $21 \mathrm{e}$ 28 dias após as aplicações (DAA) do glyphosate. Para isso, atribuíram-se notas percentuais de fitotoxicidade em relação à testemunha de acordo com a escala percentual de controle, proposta pela Asociación Latinoamericana de Malezas (ALAM, 1974).

Tabela 1. Volumes de calda $\left(\mathrm{L} \mathrm{ha}^{-1}\right)$ empregados na aplicação do glyphosate proporcionados pelas velocidades e faixa útil de aplicação do pulverizador centrífugo

\begin{tabular}{|c|c|c|c|c|c|c|c|}
\hline \multirow[t]{2}{*}{$\begin{array}{l}\text { Rotação } \\
\left.(\operatorname{rad~s})^{-1}\right)\end{array}$} & \multirow[t]{2}{*}{$\begin{array}{l}\text { Faixa útil de } \\
\text { aplicação (m) }\end{array}$} & \multicolumn{6}{|c|}{$\begin{array}{c}\text { Velocidade } \\
\left(\mathbf{k m ~ h}^{-1}\right)\end{array}$} \\
\hline & & 0,5 & 1,0 & 1,5 & 3,0 & 4,5 & 6,0 \\
\hline 105 & 1,8 & 660 & 330 & 220 & 110 & 75 & 57 \\
\hline 157 & 2,0 & 600 & 300 & 200 & 100 & 65 & 50 \\
\hline 210 & 2,2 & 550 & 270 & 180 & 90 & 60 & 45 \\
\hline 262 e 315 & 2,4 & 500 & 250 & 165 & 80 & 55 & 40 \\
\hline
\end{tabular}


Aos 30 DAA, foi determinada a porcentagem de acúmulo de massa de matéria seca das plantas com tratamento em relação às testemunhas. As plantas foram cortadas rente ao solo, identificadas, acondicionadas em saco de papel e colocadas em estufa de circulação de ar forçado, regulada a $70 \pm 1^{\circ} \mathrm{C}$, onde permaneceram durante 72 horas até atingirem massa constante. Depois, foram pesadas em balança de precisão de $0,01 \mathrm{~g}$ para posterior obtenção do percentual de acúmulo de massa de matéria seca em relação às testemunhas.

Para a análise de variância, a normalidade dos dados referentes ao acúmulo da massa de matéria seca foi analisada pelo teste de Lilliefors $(\mathrm{P}=0,01)$. Em seguida, os dados foram transformados em arcseno $\left[(\% / 100)^{1 / 2}\right]$, com a finalidade de proporcionar-lhes distribuição normal, e analisados por meio de regressão múltipla, a fim de ajustar uma equação que possibilitasse estimar cada parâmetro da tecnologia de aplicação.

A escolha dos modelos baseou-se na significância dos coeficientes de regressão e no coeficiente de determinação $\left(\mathrm{R}^{2}\right)$. Após a definição dos modelos, foram estimadas superfícies de respostas e, em seguida, realizaram-se cortes nelas, no intuito de verificar o efeito dos parâmetros com maior influência na eficácia dos tratamentos. As análises estatísticas foram realizadas no software SAEG 9.0 (Sistema de Análise Estatística e Genética - UFV), sendo as superfícies de resposta das regressões processadas no software STATISTICA 6.0.

\section{RESULTADOS E DISCUSSÃO}

Os incrementos de velocidade e rotação do pulverizador proporcionaram diminuição nos diâmetros das gotas, que variaram de 259 a $692 \mu \mathrm{m}$. Já o aumento da rotação e diminuição da velocidade, proporcionou variação de 18 a 179 gotas $\mathrm{cm}^{-2}$ na densidade de gotas e de 1,94 a 27,06 na porcentagem de cobertura (Tabela 2). A importância de um pulverizador possibilitar variar facilmente os parâmetros da aplicação reside no fato de se poder adaptálo às diferentes formas de tratamentos sem promover grandes alterações na sua estrutura ou modo de funcionamento, garantindo maior rendimento na operação.

Quando o pulverizador foi operado na velocidade de $0,5 \mathrm{~km} \mathrm{~h}^{-1}$, independentemente da rotação, o controle proporcionado pelo glyphosate foi muito ruim $(<40 \%)$, em todas as épocas de avaliações. Ademais, os efeitos fitotóxicos reduziram-se à medida que as plantas se desenvolveram. Para essas condições de operação, o DMV variou de 490 a $692 \mu \mathrm{m}$; a DEN, de 75 a 179 gotas $\mathrm{cm}^{-2}$; e a COB, de 21,39 a 27,06\%. Possivelmente, o alto DMV obtido resultou em escorrimento do produto sobre as folhas das plantas, não havendo tempo para o produto se fixar e ser absorvido. É provável que isso tenha comprometido a eficácia desses tratamentos, nos quais os volumes de calda aplicados foram superiores a $500 \mathrm{~L} \mathrm{ha}^{-1}$ (Tabela 1).

Nos tratamentos em que a variação do DMV foi de 358 a $507 \mu \mathrm{m}$, da DEN de 67 a 166 gotas $\mathrm{cm}^{-2}$ e da COB de 12,67 a $15,77 \%$, os controles foram de regular a bom (60 a 80\%) na avaliação feita aos 21 DAA. Nas avaliações realizadas aos 28 DAA, os controles chegaram a muito bons (> 81\%). Nesses tratamentos a variação do DMV, da DEN e da COB foi de 435 a $259 \mu \mathrm{m}, 53$ a 18 gotas $\mathrm{cm}^{-2}$ e 7,83 a 1,94\%, respectivamente.

Em geral, melhores controles visuais foram obtidos nos estádios mais tardios de avaliação, empregando menores diâmetros de gotas. Esse resultado concorda com aqueles obtidos por Prasad \& Cadogan (1992). A utilização da gota fina causa impactos mais suaves e, consequentemente, menores fracionamento, podendo proporcionar aumento da capacidade de aderência e redução das perdas por escorrimento sobre as superfícies das folhas.

Ademais, a atividade biológica de herbicidas sistêmicos sobre as folhas está relacionada a fatores relativos à interação físico-química da formulação e à superfície da planta. Um importante aspecto dessa interação é a quantidade da dose depositada sobre as folhas. Assim, quanto mais gotas ficarem retidas, maior dose poderá estar disponível para absorção pelas plantas e mais eficientemente será o controle (Saab et al., 2002).

Quanto ao acúmulo de massa de matéria seca de $B$. decumbens em relação às testemunhas, a análise de variância mostrou significância entre os tratamentos a 5\% de probabilidade pelo Teste F. A análise de variância dos parâmetros de regressão múltipla, indicou que a falta de ajuste das equações não foi significativa, pelo Teste $\mathrm{F}$ a $5 \%$ de probabilidade. Além disso, somente os parâmetros DEN e COB apresentaram significância pelo teste t a $1 \%$ de probabilidade. Verificou-se também que, ao retirar o parâmetro DMV da equação, o coeficiente de determinação $\left(\mathrm{R}^{2}\right)$ foi pouco alterado, indicando que ele possuía menor importância na explicação do fenômeno. O mesmo efeito não foi verificado quando se retirou da equação qualquer outro parâmetro. Isso indica que o tamanho da gota formada durante a pulverização não afeta diretamente a eficácia do glyphosate no controle de $B$. decumbens, mas a distribuição populacional das gotas sobre as folhas das plantas é determinante na eficácia da aplicação. Assim, a equação que melhor representa os efeitos observados foi ajustada em função dos parâmetros densidade de gotas e porcentagem de cobertura. Com isso, foi possível estimar a eficácia de controle nas faixas compreendidas entre 20 e $100 \%$ (Figura 1).

Verifica-se que maiores DEN e menores COB proporcionam maiores controles da planta daninha. Em termos práticos, isso pode indicar que a distribuição uniforme do 
herbicida sistêmico sobre as folhas resulta em maior controle das plantas.

Com a estimativa da eficácia dos tratamentos e de posse dos parâmetros DEN e COB, torna-se possível estimar também o diâmetro teórico, entendido como sendo o diâmetro necessário para distribuir as gotas sobre o alvo e proporcionar cobertura adequada visando o controle desejado das plantas daninhas (Equação 2).

$\mathrm{DT}=\left(\frac{\mathrm{COB} \times 10^{6}}{\frac{\pi}{4} \times \mathrm{DEN}}\right)^{1 / 2}$

em que;

DT $=$ diâmetro teórico, $\mu \mathrm{m}$;

$\mathrm{COB}=$ cobertura do alvo, $\% ; \mathrm{e}$

$\mathrm{DEN}=$ densidade de gotas, gotas $\mathrm{cm}^{-2}$.
Dessa forma, pode-se estimar que, para se obter um ótimo controle de $B$. decumbens (>91\%) é necessário ajustar o pulverizador de modo que ele distribua 30 gotas de $327 \mu \mathrm{m}$ por $\mathrm{cm}^{-2}$, proporcionando cobertura do alvo de $2,51 \%$.

Percentuais de controles mais elevados poderão ser obtidos quando se aumenta a densidade de gotas, consequentemente, reduzindo-se o diâmetro teórico. Esses resultados confirmam aqueles obtidos nas avaliações visuais. É provável que estes efeitos possam ter sido favorecido pela anatomia das folhas da espécie em estudo. Essas se caracterizam por serem estreitas e compridas, posicionando-se de modo aproximadamente paralelo ao trajeto de deposição das gotas de pulverização. Assim, acredita-se que as gotas maiores podem ter escorrido rapidamente da superfície das folhas, comprometendo a absorção do herbicida.

Tabela 2. Controles visuais de Brachiaria decumbens proporcionados por diferentes parâmetros da tecnologia de aplicação de glyphosate aos 20 dias após a emergência, na dose de $150 \mathrm{~L} \mathrm{ha}^{-1}$, obtidos por diferentes velocidades e rotações do pulverizador centrífugo

\begin{tabular}{|c|c|c|c|c|c|c|c|c|}
\hline \multicolumn{2}{|c|}{ Tratamentos } & \multicolumn{3}{|c|}{ Parâmetros da aplicação } & \multicolumn{4}{|c|}{ Controle (\%) DAA ${ }^{1}$} \\
\hline $\begin{array}{l}\text { Velocidade } \\
\left(\mathbf{k m ~ h}^{-1}\right)\end{array}$ & $\begin{array}{l}\text { Rotação } \\
\left.(\operatorname{rad~s})^{-1}\right)\end{array}$ & $\begin{array}{c}\text { DMV }^{2} \\
(\mu \mathrm{m})\end{array}$ & $\begin{array}{c}\mathrm{DEN}^{3} \\
\left(\operatorname{gotas}_{\left.\mathrm{cm}^{-2}\right)}\right.\end{array}$ & $\begin{array}{c}\mathrm{COB}^{4} \\
(\%)\end{array}$ & 7 & 14 & 21 & 28 \\
\hline \multirow[t]{5}{*}{0,5} & 105 & 692 & 75 & 21,39 & 29 & 24 & 17 & 13 \\
\hline & 157 & 573 & 95 & 23,18 & 27 & 38 & 40 & 32 \\
\hline & 210 & 504 & 119 & 24,22 & 22 & 27 & 28 & 32 \\
\hline & 262 & 492 & 152 & 25,64 & 29 & 43 & 43 & 41 \\
\hline & 315 & 490 & 179 & 27,06 & 24 & 37 & 38 & 43 \\
\hline \multirow[t]{5}{*}{1,0} & 105 & 507 & 67 & 12,67 & 25 & 66 & 65 & 75 \\
\hline & 157 & 424 & 73 & 12,89 & 24 & 58 & 70 & 83 \\
\hline & 210 & 401 & 89 & 13,40 & 34 & 59 & 72 & 75 \\
\hline & 262 & 379 & 146 & 14,68 & 18 & 62 & 66 & 75 \\
\hline & 315 & 358 & 166 & 15,77 & 27 & 77 & 72 & 82 \\
\hline \multirow[t]{5}{*}{$\overline{1,5}$} & 105 & 435 & 53 & 7,83 & 24 & 81 & 80 & 95 \\
\hline & 157 & 360 & 63 & 8,24 & 31 & 92 & 97 & 100 \\
\hline & 210 & 338 & 89 & 8,50 & 22 & 83 & 81 & 100 \\
\hline & 262 & 313 & 124 & 9,03 & 29 & 90 & 95 & 100 \\
\hline & 315 & 311 & 129 & 9,85 & 22 & 89 & 99 & 100 \\
\hline \multirow[t]{5}{*}{$\overline{3,0}$} & 105 & 431 & 25 & 4,43 & 32 & 100 & 98 & 99 \\
\hline & 157 & 340 & 33 & 4,38 & 28 & 100 & 100 & 100 \\
\hline & 210 & 305 & 51 & 4,98 & 42 & 100 & 100 & 100 \\
\hline & 262 & 297 & 73 & 5,86 & 30 & 100 & 100 & 100 \\
\hline & 315 & 268 & 75 & 5,48 & 27 & 100 & 100 & 100 \\
\hline \multirow[t]{5}{*}{$\overline{4,5}$} & 105 & 425 & 20 & 2,86 & 32 & 98 & 98 & 100 \\
\hline & 157 & 333 & 27 & 2,95 & 25 & 96 & 91 & 100 \\
\hline & 210 & 292 & 35 & 3,07 & 22 & 97 & 98 & 100 \\
\hline & 262 & 302 & 44 & 3,44 & 32 & 99 & 100 & 100 \\
\hline & 315 & 262 & 60 & 3,48 & 38 & 100 & 100 & 100 \\
\hline \multirow[t]{5}{*}{$\overline{6,0}$} & 105 & 390 & 18 & 1,94 & 31 & 98 & 100 & 100 \\
\hline & 157 & 329 & 20 & 2,09 & 26 & 96 & 99 & 100 \\
\hline & 210 & 285 & 33 & 2,62 & 26 & 100 & 100 & 100 \\
\hline & 262 & 274 & 37 & 2,53 & 28 & 98 & 100 & 100 \\
\hline & 315 & 259 & 52 & 2,92 & 35 & 97 & 100 & 100 \\
\hline
\end{tabular}

${ }^{1}$ Dias após a aplicação; ${ }^{2}$ Diâmetro da mediana volumétrica; ${ }^{3}$ Densidade de gotas; ${ }^{4}$ Cobertura. 


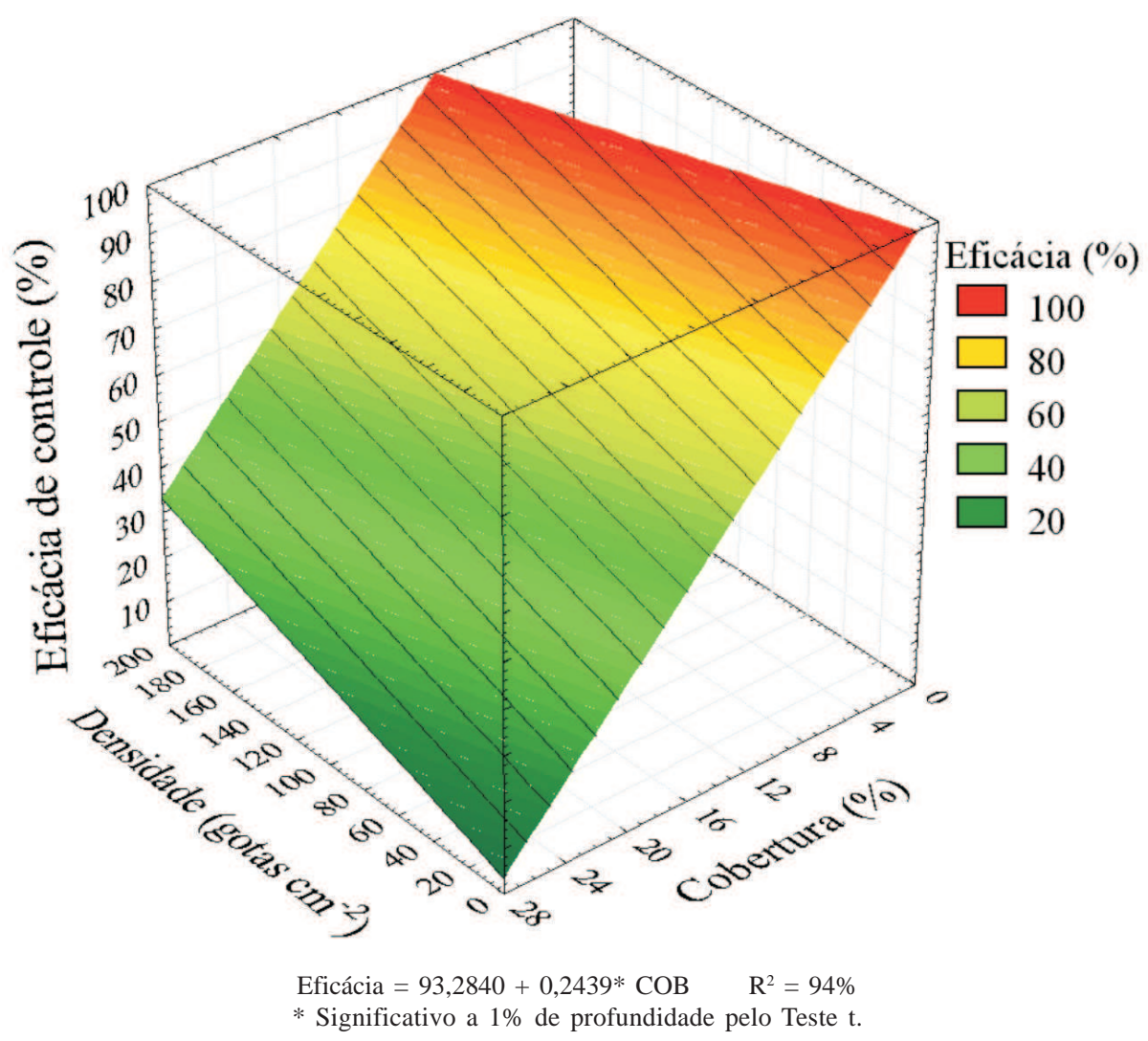

Figura 1. Eficácia do glyphosate aplicado na dose $150 \mathrm{~g} \mathrm{ha}^{-1}$ aos 20 dias após a emergência, em diferentes densidades de gotas e porcentagens de cobertura do alvo, no controle de Brachiaria decumbens.

\section{CONCLUSÕES}

Parâmetros da tecnologia de aplicação de glyphosate influenciam no controle de Brachiaria decumbens.

Volumes de calda mais baixos proporcionam melhor controle de B. decumbens.

Melhor controle de $B$. decumbens é obtido quando se realiza a aplicação de glyphosate com menores diâmetros de gotas, distribuídas mais uniformemente sobre as folhas das plantas.

\section{AGRADECIMEMTOS}

à FAPEMIG, pelo apoio financeiro a esta pesquisa.

\section{REFERÊNCIAS}

ALAM - Asociación Latino Americano de Malezas (1974) Recomendaciones sobre unificación de los sistemas de evaluacion en ensayos de control de malezas. ALAM, 1: 35-38.

Costa NV, Rodrigues ACP, Martins D, Cardoso LA \& Silva JIC (2008) Efeito de pontas de pulverização na deposição e na dessecação em plantas de Brachiaria brizantha. Planta Daninha, 26: 923-933.

Gebhardt MR, Webber CL \& Bouse LF (1986) Comparison of a rotary atomizer to a fan nozzle for herbicide aplication. Transaction American Society. Agriculture Engineering, 28:382385 .
Laguna A (2000) Maquinaria agrícola. Construcción, funcionamiento, regulaciones y cuidados. Madrid, Ed. MundiPrensa. 361p.

Liu SH, Campbell RA, Studens JA \& Wagner RG (1996) Absorption and translocation of glyphosate in aspen (Populus temuloides Michx.) as influenced by droplet size, droplet number, and herbicide concentration. Weed Science, 44:482-488.

Merritt CR (1982) The influence of form of deposit on the phytotoxicity of MCPA, paraquat and glyphosate applied as individual drops. Annals Applied Biology, 101:527-532.

Mirales A \& Hewitt AJ (1997) The international (BCPC) spray pulverization system including a drift potential factor. In: Brighton Crop Protection Conference Weeds, Brighton. Proceedings...Surrey, British Crop Protection Council. p.371380 .

Prasad R \& Cadogan BL (1992) Influence of droplet size and density on phitotoxicity of three herbicides. Weed Technology, 6:415-423.

Prat MIH, Teixeira MM \& Rodrigues GJ (2006) Influencia de la velocidade del pulverizador y la presión de líquido en la deposición del espectro de gotas. Revista Ciencia Técnicas Agropecuárias, 15:34-38.

Saab OJGA, Antuniassi UR, Fonseca ICB, Genta W \& Batistela M (2002) Efeito do tamanho de gota e volume de aplicação na deposição de agrotóxicos em folhas de videiras. Ciências Agrárias, 23:221-228.

Venturelli L, Tesouro O, Masiá G \& Fuica A (2006) Cobertura y respuesta biológica del glyphosate ante la utilización de distintas pastillas de pulverización. In: XXXV Congresso Brasileiro de Engenharia Agrícola, João Pessoa. Anais. p.67-68.

Rev. Ceres, Viçosa, v. 58, n.3, p. 299-304, mai/jun, 2011 